Absalan M, Afzal Zadeh A, Sharifi D, Khadem A A, Ghandi D, Zenouri A G H

Abass Askar K, Moody A J, Kudi A C

$177,184,185,224$

Abdi Ghezeljeh E, Danesh Mesgaran M, Vakili A R, Nassiri Moghaddam H

Afdal M, Azhar K, Alimon Razak A, Norhani A

Aikman P C, Brown R H, Gibson T M, Kliem K E, Givens D I

Alarcon P, Velasova M, Stärk K D C, Pfeiffer D U, Werling D, Wieland B

Aldai, Dugan M E R, Kramer J K G, Aalhus J L, Mantecón A R, McKinnon J J, McAllister T A 141

Alijani S, Jasoori M, Pirani N, Sayadnejad M B

Amaro P, Maia M R G, Dewhurst R J, Fonseca A J M, Cabrita A R J

Annett R W, Carson A F

Ashworth C J, Dwyer C M, Hogg C O, Etherington S L, Itani N, Landers K, Rooke J A 65

Athanasiadou S, Houdijk J G M, Eckersall P D, Low C L, Kyriazakis I 109

$\begin{array}{lr}\text { Attoh-Kotoku V, Osafo E L K } & 169\end{array}$

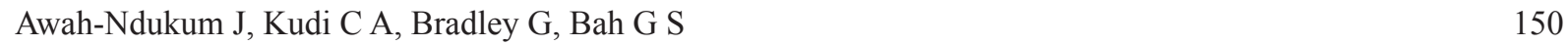

$\begin{array}{lr}\text { Azarfar Arash, Yu Peiqiang } & 83\end{array}$

Azarfar Arash, Yu Peiqiang $\quad 87$

Bahri Binabaj F, Farhangfar H, Rowlinson P, Faraji Arogh H 216

Banos G, Wall E, Mrode R, Coffey M P $\quad 51$

$\begin{array}{lr}\text { Barrier A C, Haskell M J } & 7\end{array}$

Barrier A C, Macrae A I, Haskell M J 208

Batchelor C, Main D C J, Dale V, McKeegan D E F 42

Batchelor C, McKeegan D E F 43

Bayeriyar M, Aslaminejad A A, Farajiarogh H, Bahri Beinabaj F, Danesh Mesgaran M 213

Belanche A, Martín García A I, Moorby J M, Newbold C J 170

$\begin{array}{ll}\text { Bell A } & 235\end{array}$

Bermingham M L, Handel I G, Glass E J, Woolliams J A, de Clare Bronsvoort B M, Skuce R A, Allen A R, 34 McDowell S W J, McBride S H, Bishop S C

Birch R, Edwards J, Worgan H, Macias B, Rees Stevens P, Newbold C, Nash D 176

$\begin{array}{lr}\text { Blundell R, Adam F } & 124\end{array}$

$\begin{array}{ll}\text { Bonilla-Santiago R, Nally J } & 116\end{array}$

Boyle A R, Ferris C P, O’Connell N E $\quad 94$

$\begin{array}{lr}\text { Brennan M L, Dean R S } & 40\end{array}$

$\begin{array}{ll}\text { Bruce A } & 33\end{array}$

$\begin{array}{ll}\text { Burton K, Hall C, Wells C, Billett E } & 20\end{array}$

Carberry C A, Kenny D A, Kelly A K, Creevey C J, Waters S M 77

Castillo-Olivares J

Cave M K, Mackenzie A M, Wilkinson R G, Sinclair L A 159

$\begin{array}{ll}\text { Chadwick W, Blunt R, Daly J } & 222\end{array}$

Chagunda M G G, Bagnall A, Bell D, Roberts D J

Chaji M, Mohammadabadi T 166

Chandran S, Brotherstone S, White I M S 16

Chaters G, Getachew M, Reid S

$\begin{array}{lr}\text { Chaudhry A S, Jabeen F } & 142\end{array}$

Chen Xiaolian, Liu Jianxin, Wang Jiakun 187

Cheng Z, Wathes D C, Abayasekara D R E, Elmes M, Kirkup S 158

Chiumia D, Chagunda M G G, Roberts D J, MacRae A I 8

Clark C, Mendl M, Murrell J 95

Clements D N, Bronsvoort M, Handel I, Querry D, Rose E, Summers K M 178

Clempson A, Pollott G E, Brickell J S, Bourne N E, Munce N, Wathes D C 50

Collett H, Wood E, Pfarrer C, Wapenaar W, Totemeyer S, Barrow P 131

Colman E, Vlaeminck, Abrahamse S, Dijkstra J, Fievez V 205

$\begin{array}{ll}\text { Cook M, Sand J } & 227\end{array}$

$\begin{array}{lr}\text { Coop T, Mills A } & 23\end{array}$

Cottney P D, Magowan, Ball M E E 107

Craigie C R, Lambe N R, Macfarlane J M, Maltin C A, Morris S T, Roehe R, Bunger L 67

Craigie C R, Maltin C A, Purchas R W, Ross D W, Bunger L, Morris S T, Roehe R 155

Crompton L A, Mills J A N, Kliem K E, Reynolds C K 75 
Dale A J, Ferris C P, McGettrick S

Daniel Z C T R, Buttery P J, Parr T, Brameld J M

Daniel Z C T R, Pouliot E, Buttery P J, Brameld J M, Parr T

Davies H E, Hancox L, Dodd C E R, Tötemeyer S, Wiseman J, Mellits K H

Doeschl-Wilson A B, Conington J, Davidson R, Roughsedge T, Hutchings M R, Villanueva B 59

Donaldson C L, Lambe N R, Macfarlane J M, McLean K A, Maltin C A, Bunger L 69

Duthie C, Pritchard T, Mrode R, Coffey M P, Wall E

Eaglen S A E, Woolliams J A, Coffey M P, Wall E

Ekeocha P, Ekeocha A, Fasola T, Afolabi K

Fakolade Patience

Fanchone A, Doreau M, Nozière $\mathrm{P}$

Fanchone A, Nozière P, Portelli J, Chauveau-Duriot B, Largeau V, Doreau M

Farhangfar H, Tarbaghan Jafari M, Rowlinson P, Bashtani M, Nasri Hassan Fathi M 219

Farrar C E, Garonna E, Kirkby N, Warner T, Wheeler-Jones CPD 26

Faryadimehr J, Khalajzadeh S, Bagher Sayyadnezhad M 193

Fearon A, Whitworth M, Moss B, Beattie A, Chau A, Gordon A, Matthews K 137

Fouladi M, Mirzaei F, Shakuri M, Tohidi A, Kargar S 171

Fox N J, White P C L, McClean C J , Marion G, Evans A, Hutchings M R 58

$\begin{array}{ll}\text { Franck T L } & 229\end{array}$

Freer B, Hollands T, Gardner D S, Mostyn A 123

French A T, Ogden R, Eland C, Hemani G, Corcoran B, Summers K M

French A T, Ogden R, Eland C, Hemani G, Pong-Wong R, Corcoran B, Summers K M

Garnsworthy P C, Craigon J, Hernandez-Medrano J H, Saunders N 74

Gelasakis A I, Arsenos G, Valergakis G E, Banos G 163

$\begin{array}{ll}\text { German A J } & 240\end{array}$

Ghafari H, Khadem A A, Rezaeian M, Afzalzadeh A, Sharifi S D 165

Ghafari H, Rezaeian M, Khadem A A, Sharifi S , Afzalzadeh A 172

Gildea S, Arkins S, Cullinane A 24

Goldmann W, Bossers A, Langeveld J, Andreoletti O, Lantier F, Barillet F, Torres J-M, Acin C, 112

Groschup M, Acutis P-L, Agrimi U, Nonno R, Sklaviadis T, Grassi J

\section{Guan L}

Hannant D

Hart K J, Mackenzie A M, Sinclair L A

Hayhurst C, Forbes A B, Williams D J L, Royal M D 17

Higgins H M, Brister S, Green M 118

Hillier K, Lovell M, Barrow P, Totemeyer S 130

Holt H, Lopez Alarcon P, Velasova M, Pfeiffer D, Wieland B 48

$\begin{array}{lr}\text { Honarvar M, Ghiasi H } & 218\end{array}$

$\begin{array}{lr}\text { Hopegood L, Collins S N, Reilly J D } & 19,21,22\end{array}$

Houdijk J G M, Baker A, Navajas E A, Richardson R I, Ross D W 139

Houston R D , Davey J W , Bishop S C, Gharbi K, Mota-Velasco J C, Tinch A E, Bron J E, Taggart J B 55

Hudson N P H, Mellanby R J, Shaw D J, Giannopoulos G M, Bell C E, Pearson G T, Phillips C A, 39

Dalziel L, Rhind S M

Hudson N P H, Rhind S M, Shaw D J, Giannopoulos G M, Bell C E, Pearson G T, Phillips C A, Mellanby R J 38

Humphrey T J

Hunter N, Foster J Bruce M

Huws S A, Kingston-Smith A H, Kim E-J, Lee M R F, Tweed J K S, Scott M B, Scollan N D 138

Hyslop J J, Ross D W, Navajas E A, Prieto N, Roehe R, Simm G 89

Imre A, Bukovinski A, Zhou X, Barrow P 226

Jafari Jafarpoor R, Danesh Mesgaran M, Gohari S, Danesh Mesgaran S, Ghaemi M R 199

Jafari Jafarpoor R, Heravi Mousavi A R, Danesh Mesgaran M, Vakili A R 198

Jahani-Azizabadi H, Danesh Mesgaran M, Vakili A R, Rezayazdi K, Heravi Moussavi A R, Motazhary S 192

$\begin{array}{ll}\text { Jepson P } & 231\end{array}$

Jiao H, Yan T, Wills D 183

Jones R M, Twomey D F, Hannon S, Errington J, Pritchard G C, Sawyer J 30

Keady T W J, Hanrahan J P

Khan M M H, Chaudhry Shakoor A $\quad 80$

Kiarie E, Owusu-Asiedu A, Simmins P H, Nyachoti C M 100

Kim E J, Richardson R I, Gibson K, Scollan N D 90

King M, Hillier K, Barrow P, Totemeyer S 132

Kliem K E, Reynolds C K, Humphries D J, Kirkland R, Givens D I 202 
Kliem K E, Shingfield K J, Jones A K, Givens D I

Kougioumtzis A, Oikonomou G, Arsenos G, Banos G 13

Krizova L, Vesely A, Gencurova V 156

$\begin{array}{ll}\text { Lashkari S, Taghizadeh A } & 186\end{array}$

$\begin{array}{lr}\text { Law R, McGettrick S, Ferris C } & 5\end{array}$

$\begin{array}{lr}\text { LeMoine A, Boyle R D, Miller H M } & 106\end{array}$

Lipschutz-Powell D , Woolliams J A, Bijma P, Doeschl-Wilson A B 60

$\begin{array}{lr}\text { Lückstädt C } & 145\end{array}$

$\begin{array}{lr}\text { Lynch C O, Hanrahan J P } & 160\end{array}$

$\begin{array}{lr}\text { Lynch C O, Kenny D A, Diskin M G } & 203\end{array}$

Macfarlane J M, Lambe N R, McLean K A, Wolf B T, Haresign W, Bünger L 62

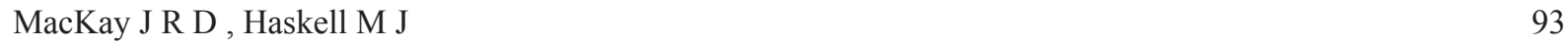

MacKintosh N D, Hart K J, Newbold C J 173

Macrae A, Burrough E, Forrest J, Parkin T, McCulloch E, Eckersall P D 31

$\begin{array}{lr}\text { Magowan E, Ball M E E } & 103,104\end{array}$

Maia M R G, Correia C A S, Alves S P, Fonseca A J M, Cabrita A R J 140

Main D C J, Whay H R

Mansor R, Burgess K, Barrett D, Whitfield P, Eckersall D $\quad 32$

$\begin{array}{lr}\text { Marchesi J } & 237\end{array}$

Marley C L, Fychan R, Davies J W, Sanderson R, Genever E, Forbes A B 180

$\begin{array}{lr}\text { Marsh S P } & 82\end{array}$

Marsh S P, Bletcher T H, Vickers M $\quad 84$

$\begin{array}{lr}\text { Marsh S P, Boyd D T } & 154\end{array}$

$\begin{array}{lr}\text { Marsh S P, Lingham T } & 85\end{array}$

$\begin{array}{lr}\text { Marsh S P, Manley C W, Wynn R } & 153\end{array}$

Masoudi A, Chaji M, Bojarpour M, Albomohsen H, Rouzegar S Rahimnahal S 189

Masri A Y, Macfarlane J M, Lambe N R, Haresign W, Bunger L 66

Mather A E, Sischo W M, Haydon D T, Mellor D J, Reid S W J 37

McCartney C A Dewhurst R J Evershed R P Pancost R D Bull I D 182

McLaren A Lambe N R ,Brotherstone S Conington J Mrode R Bünger L 64

Mitchell M A, Kettlewell P J, Villaroel M, Barreiro P, Farish M $\quad 105$

Modarresi J, Bashtani M, Fazaeli A R, Farhangfar H, Rowlinson P, Ashrafi Gol M 147

Modarresi J, Fathi Nasri M H, Dayyani O, Rashidi L, Mesgaran Danesh M, Farhangfar H 181

$\begin{array}{lr}\text { Mohammadabadi T, Chaji M } & 190\end{array}$

Mohammadi H, Shahrebabak Moradi M, Sadeghi M 215

$\begin{array}{lr}\text { Moore K, Mclean K, Bunger L } & 10\end{array}$

Moss B W, Whitworth M, Gordon A J, Chau A, Matthews K, Fearon A 136

Moss B, Fearon A, Whitworth M, Chau A, Tolland E, Devlin D, Tollerton E, Gordon A, Matthews K 143

$\begin{array}{lr}\text { Mulder I E } & 239\end{array}$

$\begin{array}{lr}\text { Mullen M P, Hanrahan J P } & 212\end{array}$

Mundy R, Hollands T, Piercy R, Verheyen K, Salonen L 157

Nasrollahi S M, Khorvash M, Ghorbani G R $\quad 195$

Nath M, Innocent G T, Gunn G J, McKendrick I J

Nelli Rahul K, Kuchipudi Suresh V, White Gavin A, Dunham Stephen P, Brown Ian H, Chang Kin-Chow 225

Nickbakhsh S, Matthews L, Reid SWJ, Kao RR 61

Nikolaou G, de Bonont M, Heitzel U 125

Oguike M A, Igwe C O, Odika I E, Onyekweodiri E O 179

$\begin{array}{lr}\text { O'Keeffe G E } & 191\end{array}$

Orton S, Heayns B, Martin E

\begin{tabular}{lr} 
Otter A & 115 \\
\hline
\end{tabular}

$\begin{array}{ll}\text { Patterson N, Coffey T, Werling D } & 117\end{array}$

Patterson R, Dalby P, Seyfert H-M, Werling D 174

$\begin{array}{lr}\text { Peffers M, Beynon R, Clegg P } & 29\end{array}$

Philbey A, Taylor D, Thompson H 126

Phythian C J, Cripps P J, Toft N, Michalopoulou E, Jones P H, Grove-White D H, Clarkson M J, 120

Winter A C, Duncan J S

Plinston C, Hart P, Chong A, Hunter N, Foster J, Piccardo P, Manson JC, Barron RM 111

$\begin{array}{lr}\text { Pollott G E } & 194\end{array}$

$\begin{array}{lr}\text { Pollott G E, Baber P L } & 211\end{array}$

$\begin{array}{ll}\text { Pollott G E, Wall E, Coffey M P } & 78\end{array}$

Pollott G E, Wathes D C, Brickell J S, Clempson A M, Hill S 49 
Pritchard T, Coffey M, Mrode R, Moore K, Wall E 11

Pritchard T, Coffey M, Mrode R, Moore K, Wall E 209

Reader R, Morgan C, Hegarty M, McEwan N, Powell W, Nash D 175

Rezaeian M, Honarzad J, Mousavi S S 204

$\begin{array}{lr}\text { Ricci P, Waterhouse A } & 79\end{array}$

$\begin{array}{lr}\text { Richardson C } & 127\end{array}$

Robaye V, Dotreppe O, Hornick J L, Istasse L, Dufrasne I 135,149

$\begin{array}{lr}\text { Roberts C } & 234\end{array}$

Roehe R, Baxter E M, Knap P W, Lawrence A B 110

Rose M T, Cratchley T D, Pearson S 4

Ross S A, Chagunda M G G , Topp C F E, Ennos R A 76

Rowe S J, Karacaoren B, de Koning D J, Velander I, Haley C S, Archibald A L 56

Ryan K, Stewart P, Foster J, Parnham D, Bossers A, Goldmann W 114

Rymer C, Humphries D J , Blake J S , Givens D I 86

Sagatarame S, Fleming H R, Theobald V, Lee M R F 70

Sakkas P , Houdijk J G M , Athanasiadou S , Kyriazakis I 121

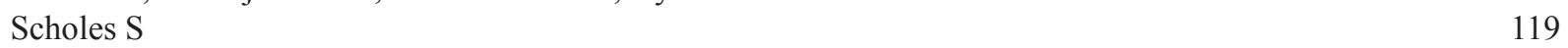

Scollan N D , Richardson R I, Kim E J 134

Scott-Browne H, Blackie N, Tibbott A, Cooke R 152

Sgwane T S , Dlamini B J , Dlamini M 161

Shirali M, Doeschl-Wilson A, Knap P W, Duthie C, Kanis E, van Arendonk J A M, Roehe R 99

Shirif A, Chaudhry A, Ben Hage K 167

Smith LA, Houdijk JGM, Kyriazakis I 98

Smith S A, Jann O C, Haig D, Werling D, Emes R D, Glass E J 128

Sofla Savar S , Javaremi Nejati A, Abbasi M A, Torshizi Vaez R, Chamani M, Dezfuli Taheri B 214

Sofla Savar S , Taheri Dezfuli B , Seyedabadi H R 220

Steele M, Crabb N P, Moore L J

Stergiadis S, Leifert C, Seal CJ, Eyre M D, Butler G 1

Stevens H, Blackie N, Amory J R 97

$\begin{array}{lr}\text { Suttle N } & 133\end{array}$

Moumen Syeed S M, Tokasi M V, Bafti Shamsaddini M, Karegar N 200

Szyszka O, Tolkamp B Edwards S, Kyriazakis I 92

Taylor A E, Jagger S, Toplis P, Wellock I, Miller H M 221

$\begin{array}{ll}\text { Terry R L, Wells D J } & 27\end{array}$

Tibbott A, Cooke R, Scott-Browne H, Blackie N 96

Todd D L, Woolliams J A, Roughsedge T R 81

Toplis P, Wellock I J, Miller H M 102

Vargas-Bello-Pérez E, Salter A M, Loor J J , Garnsworthy P C 6

Velasova M, Alarcon P, Werling D, Chang Y, Nevel A, Wieland B 46

Wall E, Coffey M Sadeghi-Sefidmazgi A, Amer P 15

Walsh A M, Sweeney T, Callan J J, Bahar B, O’Doherty J V 108

$\begin{array}{lr}\text { Weaver G, Watson M, Kaiser P } & 52\end{array}$

Wellock I J, Toplis P, Stewart A, Miller H M 101

Werkman M, Munro L A, Green D M, Murray A G, Turnbull J F 146

White G A, Dunham S P, Brookes S M, Germundsson A, Garcon F, Núñez A, Chang K-C, 45

Brown I H, Consortium COSI

White G A, Dunham S P, Nelli R K, Brown I H , Kuchipudi S V , Chang KC 223

Wilkinson J M , Garnsworthy P C , Huxley J N 230

$\begin{array}{ll}\text { Winter M } & 236\end{array}$

Witcomb LA, Green L E, Wellington E M W, Kaler J, Ul Hassan A, Calvo Bado L A, Grogono Thomas R, 122 Medley G F

Yates J , Parkin T D H , Casey M , Mellor D J , Kao R R, Boden L A 18

Zaralis K, Nadeau E, Johansson S, Helander C, Nørgaard P, Murphy M 88

Zervos A, Kasapidou E, Papaloukas L, Sinapis E 144 